\title{
Comparison of mechanisms of kinetochore capture with varying number of spindle microtubules
}

\author{
Indrani Nayak ๑, Dibyendu Das, ${ }^{*}$ and Amitabha Nandi $\odot^{\dagger}$ \\ Department of Physics, Indian Institute of Technology Bombay, Powai, Mumbai 400076, India
}

(Received 26 July 2019; accepted 3 January 2020; published 3 February 2020)

\begin{abstract}
The biophysical mechanisms of kinetochore capture by spindle microtubules within cells are stochastic processes with a moving target searched by multiple walkers inside a confined volume. We study and compare two such mechanisms: dynamic instability-driven search and capture, common in many eukaryotes, and angular diffusion of pivoted microtubules reported in fission yeast. Characteristic times associated with the rare events of capture scale as a power law with the microtubule number, and their comparison provides a physical basis for the selection of one mechanism over another.
\end{abstract}

DOI: 10.1103/PhysRevResearch.2.013114

\section{INTRODUCTION}

An essential function of a living cell is to segregate chromosomes between the two daughter cells [1]. The mother cell achieves this by forming a spindle: a microscopic structure made of cytoskeletal filaments and cross-linking proteins, stretching across its length between the poles [2,3]. One important aspect of spindle morphogenesis is the attachment of microtubules (MTs) to kinetochores (KCs) [4], which are protein complexes on the chromosomes [see Fig. 1(a)]. How does a MT attach to a KC? This question has been raised and studied before, and the mechanism by which this attachment takes place can vary across cell types [5-9]. One such mechanism, known as search-and-capture (S\&C), involves dynamically unstable MTs growing out of the centrosome in different random directions [10-13]. The KC attaches to one of the MTs when they both hit each other. S\&C has been well studied using simplified theoretical approaches [12,14-16] as well as detailed computational models [7,15,17]. Recent in vivo experiments have found that the actual capture processes may be influenced by other factors $[5,7,8,15,18-30]$ beyond simple S\&C.

The biophysical problem of KC capture by multiple MTs maps to a problem in a stochastic process where a moving target is searched by multiple diffusing entities. Furthermore, the process takes place under confinement (cellular volume). While for a static target in confined space there exist several studies in the literature [31-36], for a moving target only a few exact results are known in $1 d$ open space [37-39]. All these studies consider point-particle random walkers. On the other hand, MTs are extended objects exhibiting complex stochastic dynamics $[7,15,16]$. Therefore a

\footnotetext{
*dibyendu@phy.iitb.ac.in

†amitabha@phy.iitb.ac.in

Published by the American Physical Society under the terms of the Creative Commons Attribution 4.0 International license. Further distribution of this work must maintain attribution to the author(s) and the published article's title, journal citation, and DOI.
}

detailed study of this complex biophysical problem in relation to the above stochastic process problem motivates this work.

A second motivation is related to the spindle assembly in fission yeast [6,8,40-44], and more specifically, to an experiment [8] which reported an exception to the usual S\&C mechanism in Schizosaccharomyces pombe (fission yeast). Here a diffusing $\mathrm{KC}$ inside the nucleus is captured by MTs executing rotational diffusion (RD) $[8,40,42]$, being pivoted at the spindle pole body (SPB) [see Figs. 1(a) and 1(b)]. The pivoting motion appears during a state of pause [Fig. 1(b)] interrupting the dynamic instability of the MTs. This mechanism is driven by thermal noise, unlike $\mathrm{S} \& \mathrm{C}$, which is chemically driven. This raises an intriguing question as to why, unlike many other organisms, fission yeast prefers a passive mechanism over an active one and whether there is a physical basis for the choice.

Does the number of spindle MTs decide the choice of a capture mechanism? In fission yeast, fewer spindle MTs are available ( 3-5 MTs per KC) [8,45], unlike more complex eukaryotes (e.g., human cells have $\sim 17$ MTs per KC $[4,46]$ ). This question requires a systematic study of capture times comparing the two mechanisms (S\&C and RD) as a function of MT number $N$. Although this has been done for fixed number of MTs $[7,15,17,40]$, a study by varying $N$ for a moving $\mathrm{KC}$ is lacking, and the scaling of the capture times with $N$ is unclear. Earlier studies on capture of a static target by $N$ MTs suggested a heuristic form of the mean capture time $\sim 1 / N[15,47]$. For a static target, the characteristic time (associated with the tail of the capture time distributions) $\propto 1 / N$ exactly, as the problem simplifies to $N$ independent capture events $[36,48,49]$. However for a moving KC, which is biologically more relevant, this conclusion is no longer valid - the relative coordinates of the MTs with respect to the $\mathrm{KC}$ are correlated, thus leading to nontrivial dependence of the capture times on $N$. As commented earlier, this is analogous to a classic problem of $N$ lions chasing a moving lamb [37-39] in the stochastic process literature, albeit in confined volume. A deviation from the $1 / N$ behavior is known for a special scenario of an evasive target on a lattice which starts moving only when the predators are close by [50]. 

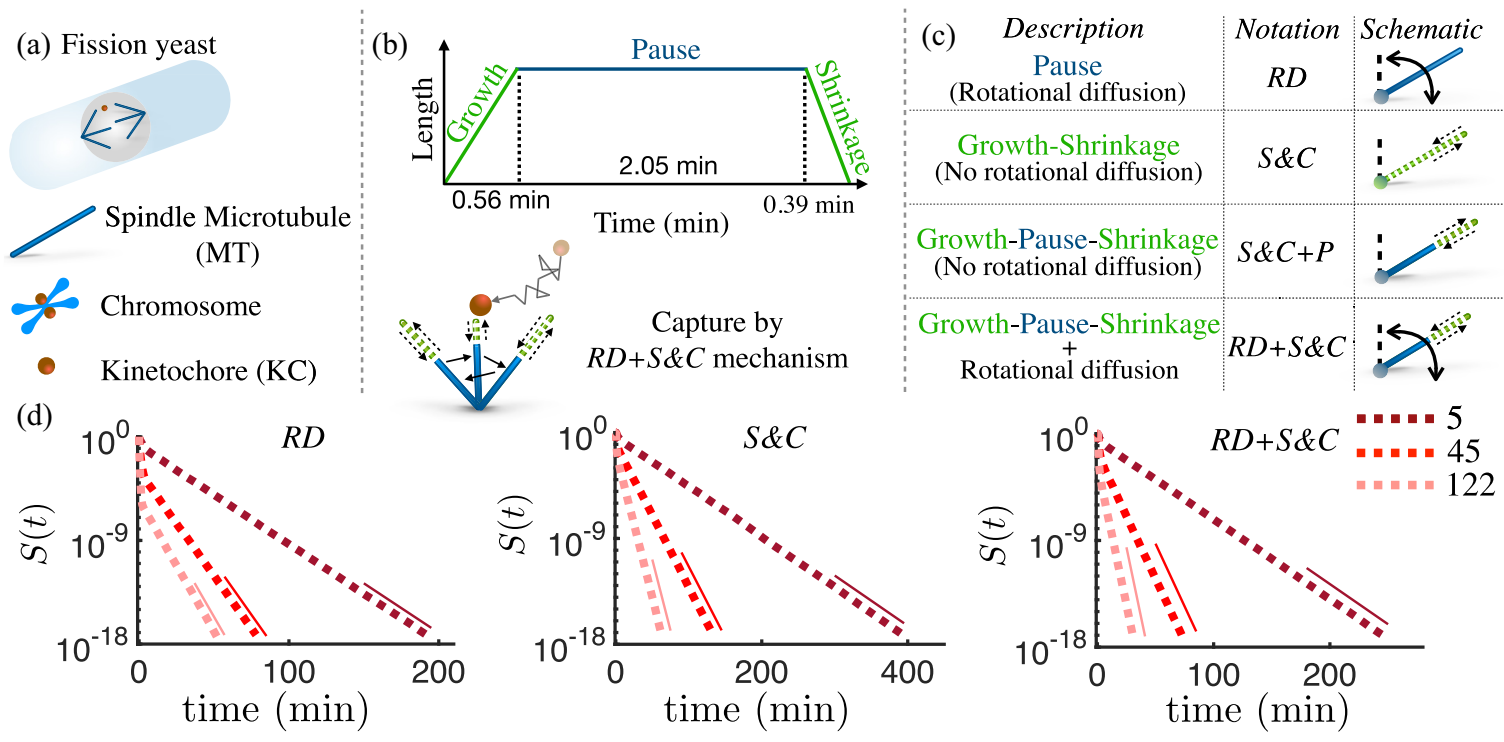

FIG. 1. (a) Schematic of a fission yeast cell. Here the spindle is formed inside the nucleus. (b) Length variation with time is schematically shown for a spindle MT (top). The timescales of growth, shrinkage, and pause states as known from experiments [8] are indicated. The schematic (below) shows the capture of a KC by a MT. (c) The four different models studied in this paper are schematically shown here. The pause state is indicated in blue, while growth and shrinkage is indicated in green. Straight and curved arrows represent the linear growth/shrinkage and angular diffusion, respectively. (d) Fraction of lost KCs or survival probability $S(t)$ curves for three different mechanisms are shown for different MT numbers $N$. Fits to the asymptotic tails are shown with solid lines. The timescales of models RD and RD+S\&C are close, while that of S\&C are comparatively larger.

In this paper we numerically study the statistics of firstpassage times $[33,39,51]$ of $\mathrm{KC}$ capture by multiple MTs and show that the characteristic times of capture $\sim N^{-\beta}$ with $\beta \neq 1$. We estimate the characteristic times using an algorithm $[52,53]$ which obtains survival probabilities of KC very precisely, as well as propose a method based on extreme value statistics [54,55]. Using this temporal measure, we demonstrate that depending on the available number of MTs, one mechanism may be preferred over another.

\section{MODELS AND METHODS}

Our primary aim here is to compare the efficiency of different capture mechanisms as a function of MT number $N$. To this end we compare four different models [Fig. 1(c) and Appendix A] to identify the relative contributions of active growth-shrinkage versus passive angular diffusion. First, we study a model [8] in which the growth-shrinkage kinetics of MTs are ignored. Preformed MTs of fixed length $L_{M T}$, pivoted at the SPB, sweep the space in search of a $\mathrm{KC}$-in this case referred to as the RD model. Similarly, one may imagine a hypothetical scenario in which the MTs in fission yeast did not have the pause state, like in many other eukaryotes, and switch directly from the growth to a shrinkage state-we will refer to this pure search-and-capture case as the S\&C model. A third model has growth-pause-shrinkage kinetics, as seen in the experiments, but during the paused state, the angular motions of MTs are suppressed-this will be referred to as the $\mathrm{S} \& \mathrm{C}+\mathrm{P}$ model. A similar model has been considered earlier in a computational work but only for $N=1$ [17]. Finally, to have a benchmark, we study the full model that incorporates all the features observed in experiments, i.e., a MT performing both RD and growth-pause-shrinkage kinetics simultaneously [see Fig. 1(b)]-we will refer to it as the RD+S\&C model. The mathematical equations of transport for the $\mathrm{KC}$ and MT and the Langevin and kinetic Monte Carlo simulation details of all these models are discussed in Appendix A; all the parameters used (Table II in Appendix A) are taken from the in vivo measurements reported in experiments [8].

In simulations we calculate the fraction of lost kinetochores or equivalently, survival probability $S(t)$ [51]. For capture processes in free space, $S(t)$ typically has power-law tails [S(t) $\sim t^{-\gamma}$, for $t \rightarrow \infty$ ] [39], while in confined geometries (like in our study, where the KC capture takes place inside the nuclear volume), it is expected to have exponential tails asymptotically: $S(t) \sim \exp (-t / \tau)$ [36,51] [Fig. 1(d)]. We are not simply interested in the mean times $\langle t\rangle=\int_{0}^{\infty} d t^{\prime} S\left(t^{\prime}\right)$ [33,51], which may have limitations (see below), but also the characteristic times $\tau$ associated with the asymptotic behavior of $S(t)$. We used two different methods to estimate $\tau$ in this paper (see Appendix B). Results presented here are for cases of lateral capture, i.e., a $\mathrm{KC}$ can attach to any point along the length of an MT body. The special cases of tip capture to the vicinity of MT tips are discussed in Appendix C.

\section{RESULTS}

We first plot the mean times of $\mathrm{KC}$ capture $\langle t\rangle$ as a function of MT number $N$ in Fig. 2 . We see that for $N \lesssim 50,\langle t\rangle_{R D}<$ $\langle t\rangle_{S \& C}$, implying that a passive RD mechanism is undoubtedly more efficient than S\&C. We see that for the RD+S\&C model, $\langle t\rangle$ is close (and marginally greater) than that of RD and distinctly smaller than that of S\&C. This hints that if the MTs suffer catastrophe (which happens in the RD+S\&C model but not in the RD model), a delay is introduced in the capture process even if they do rotational diffusion. 


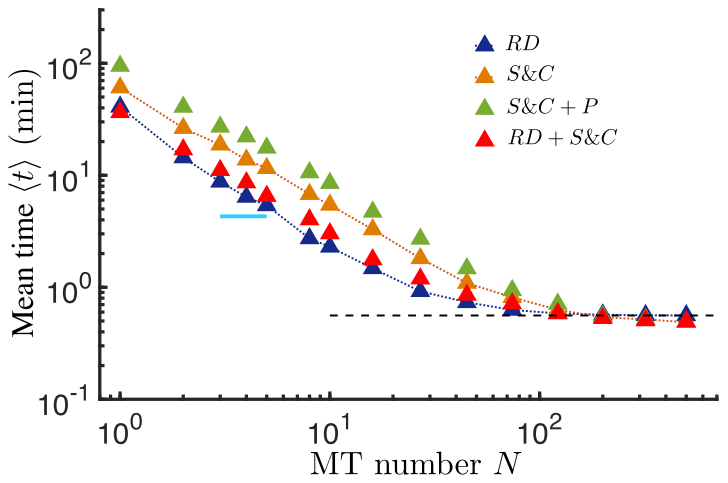

FIG. 2. Mean capture time $\langle t\rangle$ for the four models are plotted vs $N$ in log-log scale. The important cases of RD (blue) and S\&C (orange) are highlighted with symbols joined by lines. The experimental mean time $\approx 4.30 \mathrm{~min}$ is indicated by a cyan line-segment (for 3-5 MTs). At large $N$ all the $\langle t\rangle$ values saturate to a threshold $\approx 0.56 \mathrm{~min}$. Since the RD model does not take the initial growth of the MTs into account, we added $0.56 \mathrm{~min}$ to $\langle t\rangle_{R D}$ here for comparison with other models.

This trend of ascending timescales with the elimination of angular diffusion (see Table IV in Appendix D) is further confirmed as $\langle t\rangle$ is the highest for the $\mathrm{S} \& \mathrm{C}+\mathrm{P}$ model with dynamically unstable MTs along with a pause state which does not rotationally diffuse. For fission yeast, $N \sim 3-5$ MTs [8]. From the published experimental data (Fig. 1(b) in [8]) we estimated the mean capture time $\langle t\rangle \approx 4.3 \mathrm{~min}$ (cyan line in Fig. 2). This value is reasonably close to $\langle t\rangle_{R D}(\simeq 5.4 \mathrm{~min})$ and $\langle t\rangle_{R D+S \& C}(\simeq 6.5 \mathrm{~min})$ obtained from our simulations for $N=$ 5. On the other hand, the corresponding $\langle t\rangle_{S \& C}(\simeq 11.6 \mathrm{~min})$ and $\langle t\rangle_{S \& C+P}(\simeq 17.6 \mathrm{~min})$ are way larger. Thus the temporal efficiency of RD of MTs over S\&C could be a possible physical reason underlying its biological selection in cells like fission yeast with a small number of MTs. However, the puzzle still remains as to why $\mathrm{S} \& \mathrm{C}$ is ubiquitous in the eukaryotic world, particularly in cells with larger number of spindle MTs. At large $N$ in Fig. 2, we see that the $\langle t\rangle$ for all the models converges to the limit $\approx 0.56 \mathrm{~min}$, which is the mean growth time of an MT from the SPB to its average length $L_{M T}$. Thus the statistical differences of the different models, if any, cannot be captured by these mean values.

The mean value is not sufficient to represent the full statistics of a $\mathrm{KC}$ capture process, as is often the case with other stochastic problems [32,34]. First, the $S(t)$ function depends strongly on the initial spatial distribution of the $\mathrm{KC}$ and MTs. For our simulations, we use a generic initial condition, namely, the $N$ MTs are initially oriented in random directions. In Fig. 3(a) we show that, if instead, the MTs are initially oriented in special directions, the $S(t)$ curves are all distinct - each one of them would correspond to distinct mean values [area under the $S(t)$ curves]. Second, from Fig. 3(a) it is clear that $S(t)$ curves quite generically are not single exponentials. As a result, $\langle t\rangle \neq \tau$, and we quantitatively show this departure for all four models as a function of $N$ (Appendix E). The presence of multiple well-separated timescales [see inset of Fig. 3(a)] thus makes a single mean value somewhat misleading [56-59]. We study a quantity called the uniformity
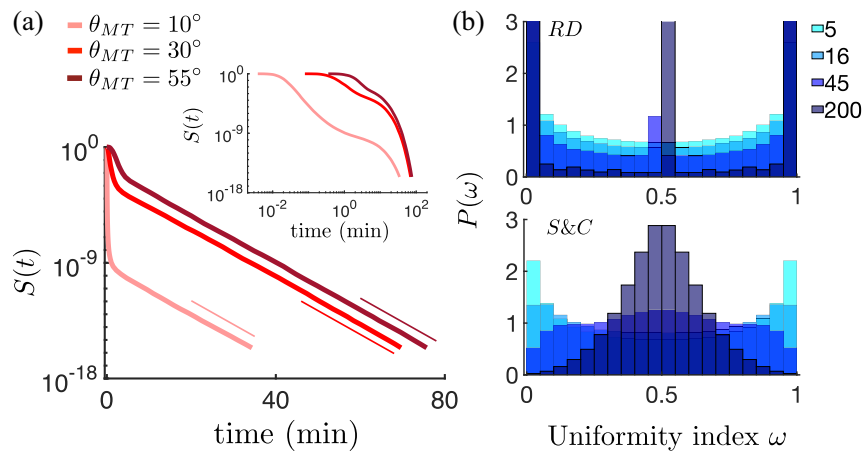

FIG. 3. (a) Semilog plot of $S(t)$ vs time $t$ for the RD mechanism with $N=45$ MTs for three different initial orientations $\theta_{M T}$ (with random $\phi_{M T}$ and $\theta_{K C}=0$ ) at $t=0$. The tails of the curves are parallel (as indicated by the fits), implying a common characteristic time $\tau$. Inset shows the presence of multiple timescales in a loglog plot. (b) $P(\omega)$ vs $\omega$ are shown for RD and S\&C models for different $N$.

index $\omega=t_{1} /\left(t_{1}+t_{2}\right)$ [59], where $t_{1}$ and $t_{2}$ are two random instants of $\mathrm{KC}$ capture times. If $t_{1} \approx t_{2}$, it implies $\omega \approx 0.5$, while if $t_{1}$ and $t_{2}$ are very different, $\omega$ is close to 0 or 1 . The distribution function $P(\omega)$ is accordingly either unimodal (around $\omega=0.5$ ) or multimodal. Since we are interested in the extent of temporal variations of capture times over and above the common mean growth time, in each model we subtract this offset and use the samples to construct $P(\omega)$ as defined above for different $N$. In Fig. 3(b), while for the RD model $P(\omega)$ stays multimodal with varying $N$, for the $\mathrm{S} \& \mathrm{C}$ model it is multimodal at small $N$ and becomes unimodal at large $N$. For RD, the MTs explore the $3 d$ nuclear volume using pivoting; as a result the diversity of capture times of the diffusing KC due to both long and short trajectories persist for any $N$. On the other hand, for S\&C, MTs move rectilinearly in $3 d$ space. They appear as static line traps in the path of the $\mathrm{KC}$, and thus with increasing $N$, the number of long trajectories decreases (due to a rise in the number of traps), making $P(\omega)$ unimodal [Fig. 3(b)].

A strong dependence of $S(t)$ on initial conditions [Fig. 3(a)] implies that even variance of the timescales will be initial condition dependent, like the mean. Yet the most robust quantity is the characteristic time $\tau$-all the curves in Fig. 3(a) have the same $\tau$. Physically this happens as $\tau$ represents timescales of rare events of $\mathrm{KC}$ capture involving long trajectories of motion which remove memories of initial conditions $[31,35]$. Biologically, too extreme times are important to estimate upper bounds for completion of the KC capture process, which affects mitosis. Thus for both theoretical robustness and practical implications, we focus on this timescale in the rest of the paper. To calculate $\tau$, we use an algorithm to study the first passage of an ensemble $(\sim 1000$ copies) of our MT-KC systems. Once a part of the ensemble attains first passage, the original ensemble size is restored by cloning the copies which survive up to that time [52,53] (see method 1 in Appendix B1). Performing this step repeatedly we obtain $S(t)$ up to precisions $\sim 10^{-17}$ [Fig. 1(d)], which cannot be achieved by conventional algorithms.

We compare $\tau$ for RD and S\&C models in Fig. 4. When $N$ is small, we see that $\tau_{R D}<\tau_{S \& C}$, implying better efficiency 


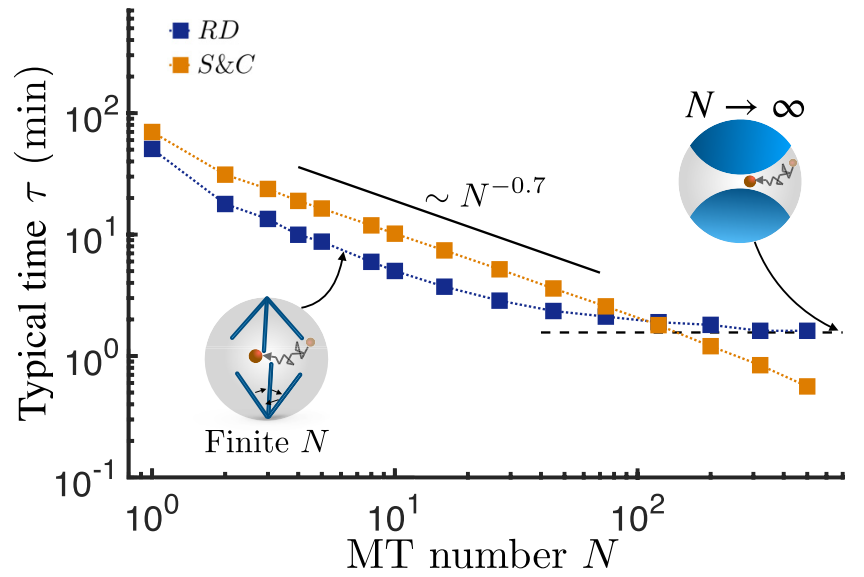

FIG. 4. $\tau$ vs $N$ for the RD (blue) and S\&C (orange) models. A power-law fit of $\tau_{S \& C}$ is shown. Two schematics for the RD model with finite $N$ and $N \rightarrow \infty$ limits are shown. The limiting value of $\tau_{R D}^{\text {sat }} \approx 1.56 \mathrm{~min}$ is shown by a dashed line in the $N \rightarrow \infty$ limit, obtained by simulating the $\mathrm{KC}$ diffusion in the presence of two curved absorbing surfaces.

of the RD model over $\mathrm{S} \& \mathrm{C}$ - a conclusion similar to that obtained using $\langle t\rangle$ (see Appendix D). On the other hand, as $N$ increases, $\tau_{R D}$ starts saturating for $N \gtrsim 20$, while $\tau_{S \& C}$ continues to decrease monotonically. This leads to a crossing of the two curves. Study of $\tau$ thus suggests that the $\mathrm{S} \& \mathrm{C}$ mechanism is more advantageous in cells with a large number of MTs, rationalizing its prevalence in eukaryotes $[3,7,46]$. For a comparison of the four different models, see Appendix C.

The saturation seen in $\tau_{R D}$ is due to geometrical constraints in the RD model. The MT tips diffuse on a spherical surface of radius $L_{M T}, \theta_{M T} \in\left[0, \theta_{M T}^{\max }\right]$ with $\theta_{M T}^{\max }=\cos ^{-1}\left(L_{M T} / 2 R\right)$, and $\phi_{M T} \in[0,2 \pi]$. There are two such surfaces spanned by MTs pivoted at the SPBs. As $N$ becomes large, the entire volume below (and above) these surfaces becomes inaccessible to the $\mathrm{KC}$, since they are instantaneously captured there. We performed a separate simulation with the initial position of the $\mathrm{KC}$ taken around the equatorial plane between two curved absorbing surfaces. The $\tau_{R D}^{\text {sat }} \approx 1.56 \mathrm{~min}$, obtained from this $N \rightarrow \infty$ limit simulation, is shown by a dashed line in Fig. 4 . The values of $\tau_{R D}$ converge to $\tau_{R D}^{\text {sat }}$ for large $N$, as expected. To further confirm that the saturation of $\tau$ with increasing $N$ is a generic phenomenon due to the existence of a continuous absorbing surface inside the confining volume, we performed an analytical calculation for a toy model (see Appendix F).

In Fig. 4 we observe that for $\mathrm{S} \& \mathrm{C}$ the characteristic time shows a nontrivial power-law decay $\tau_{S \& C} \sim N^{-\beta}$ with $\beta \approx 0.7$. This is a striking deviation from the known results with a static target where $\tau \sim 1 / N[36,48,49]$. As noted earlier, this arises due to the correlated movement of the MTs relative to the moving KC. From our unpublished results on the lion-lamb problem under confinement [60], we know that the exponent $\beta$ may depend on various factors such as diffusivity and geometrical constraints.

While method 1 estimates $\tau$ precisely given adequate computational resources, it may not be useful to experimentalists with a limited number of measured samples. We propose a
TABLE I. Method 2 compared to method 1.

\begin{tabular}{|c|c|c|c|c|c|c|}
\hline \multirow[b]{3}{*}{$N$} & \multirow[b]{3}{*}{ Method 1} & \multicolumn{2}{|l|}{$\tau_{R D}(\min )$} & \multicolumn{3}{|c|}{$\tau_{S \& C}(\min )$} \\
\hline & & \multicolumn{2}{|c|}{$\begin{array}{c}\text { Method } 2 \\
N_{1}=1000\end{array}$} & \multirow[b]{2}{*}{ Method 1} & \multicolumn{2}{|c|}{$\begin{array}{l}\text { Method } 2 \\
N_{1}=100\end{array}$} \\
\hline & & $N_{2}=10$ & $N_{2}=100$ & & $N_{2}=10$ & $N_{2}=100$ \\
\hline 5 & 8.75 & 9.62 & 8.58 & 16.39 & 12.24 & 17.97 \\
\hline 16 & 3.72 & 3.81 & 3.77 & 7.41 & 3.69 & 6.96 \\
\hline 27 & 2.86 & 2.91 & 2.84 & 5.18 & 4.97 & 5.43 \\
\hline 45 & 2.35 & 1.75 & 1.51 & 3.60 & 3.39 & 3.76 \\
\hline
\end{tabular}

method (see method 2 in Appendix B2) based on extreme value statistics [54,55], which allows a rough estimate of $\tau$ from limited available data. If the experiment measures $N_{t}$ random capture times, those may be divided into $N_{2}$ sets, each containing $N_{1}=N_{t} / N_{2}$ samples. The variance $\sigma^{2}$ of the maximum times $t_{\max }$, drawn from each of these $N_{2}$ sets, is related to the characteristic time as $\tau=\sqrt{6} \sigma / \pi$ (see Appendix B2). In Table I, we see that for S\&C, for just $N_{1}=100$ and $N_{2}=10$, an experimentalist may estimate $\tau$ close to the actual values. For $\mathrm{RD}$, convergence to $\tau$ requires a relatively larger sample set.

\section{CONCLUSIONS}

Finding KCs by the spindle MTs is a crucial part of mitosis. Why do organisms often select different mechanisms to achieve capture? It is a moot question whether the existence of any specific mechanism is just a random selection or, given the machinery and resources available to an organism, the chosen mechanism optimizes something. A systematic study of first-capture times as a function of MT number $N$ comparing different possible mechanisms is lacking. In this paper, we address this question by studying the temporal optimality of different mechanisms. Interestingly we find that one mechanism may be chosen over another, depending on the number of spindle microtubules. We find that $\mathrm{RD}$ is more efficient than S\&C only when the MTs are fewer in number, which might be an advantage for organisms such as fission yeast. Our study shows that the mean capture time of $\approx 4.3 \mathrm{~min}$ as seen in experiments cannot be explained by a pure search-and-capture mechanism but instead by pivoting. On the other hand, $\tau_{R D}$ tends to saturate beyond $N \gtrsim 20$, while $\tau_{S \& C}$ decreases monotonically, rendering the S\&C mechanism more efficient than RD for large $N$-this may explain the common occurrence of $\mathrm{S} \& \mathrm{C}$ among eukaryotes. For a few MTs, RD helps them explore a larger solid angular space than $\mathrm{S} \& \mathrm{C}$ in a fixed time. In the latter case, misoriented MTs and their times of growth-shrinkage may both lead to additional delays in the first encounter with the $\mathrm{KC}$.

The MT-KC capture problem has a capture time distribution with an exponential tail due to confinement. Finding $\tau$ is challenging as it associated with rare events. We used an algorithm based on successive cloning of copies of the system and obtained $S(t)$ to very high precision, leading to an accurate estimate of $\tau$. Furthermore, we showed that an approximate estimate of $\tau$ is possible using the theory of extreme value statistics. These techniques are quite general 
TABLE II. Parameter values used in simulation.

\begin{tabular}{|c|c|c|}
\hline Models & Parameters & Values \\
\hline $\mathrm{RD}$ & $\begin{array}{l}\text { Radius of nuclear sphere }(R) \\
\text { Radius of } \operatorname{KC}(a) \\
\text { Length of } \operatorname{MT}\left(L_{M T}\right) \\
D_{K C} \\
D_{M T} \text { (angular diffusion coefficient) }\end{array}$ & $\begin{array}{l}1.55 \mu \mathrm{m} \\
0.124 \mu \mathrm{m} \\
1.5 \mu \mathrm{m} \\
0.0354 \mu \mathrm{m}^{2} \mathrm{~min}^{-1} \\
0.0603 \mathrm{rad}^{2} \mathrm{~min}^{-1}\end{array}$ \\
\hline Dynamic instability & $\begin{array}{l}D_{K C} \\
\text { Tubulin subunit length } \\
\text { Growth rate }\left(r_{+}\right) \\
\text {Shrinkage rate }\left(r_{-}\right)\end{array}$ & $\begin{array}{l}0.0354 \mu \mathrm{m}^{2} \min ^{-1} \\
6.15 \times 10^{-4} \mu \mathrm{m} \\
4387.5 \text { subunit } \min ^{-1} \\
6175 \text { subunit } \min ^{-1}\end{array}$ \\
\hline $\mathrm{S} \& \mathrm{C}$ & $\begin{array}{l}\mathbf{G} \text { to } \mathbf{S} \text { frequency }\left(f_{+-}\right) \\
\mathbf{S} \text { to } \mathbf{G} \text { frequency }\left(f_{-+}\right)\end{array}$ & $\begin{array}{l}1.8 \min ^{-1} \\
0 \min ^{-1}\end{array}$ \\
\hline $\mathrm{S} \& \mathrm{C}+\mathrm{P}$ & $\begin{array}{l}\mathbf{G} \text { to } \mathbf{P} \text { frequency }\left(f_{+0}\right) \\
\mathbf{P} \text { to } \mathbf{S} \text { frequency }\left(f_{0-}\right) \\
\mathbf{P} \text { to } \mathbf{G} \text { frequency }\left(f_{0+}\right) \\
\mathbf{S} \text { to } \mathbf{P} \text { frequency }\left(f_{-0}\right)\end{array}$ & $\begin{array}{l}1.8 \min ^{-1} \\
0.49 \min ^{-1} \\
0 \min ^{-1} \\
0 \min ^{-1}\end{array}$ \\
\hline $\mathrm{RD}+\mathrm{S} \& \mathrm{C}$ & $\begin{array}{l}D_{0} \\
\tilde{D}_{M T} \\
\mathbf{G} \text { to } \mathbf{P} \text { frequency }\left(f_{+0}\right) \\
\mathbf{P} \text { to } \mathbf{S} \text { frequency }\left(f_{0-}\right) \\
\mathbf{P} \text { to } \mathbf{G} \text { frequency }\left(f_{0+}\right) \\
\mathbf{S} \text { to } \mathbf{P} \text { frequency }\left(f_{-0}\right)\end{array}$ & $\begin{array}{l}0.2036 \mathrm{rad}^{2} \min ^{-1} \mu \mathrm{m}^{3} \\
D_{0} / r_{M T}^{3}\left(\text { if } r_{M T}>=1.5 \mu \mathrm{m}\right), \\
0.0603 \operatorname{rad}^{2} \min ^{-1}\left(\text { if } r_{M T}<1.5 \mu \mathrm{m}\right) \\
1.8 \mathrm{~min}^{-1} \\
0.49 \mathrm{~min}^{-1} \\
0 \mathrm{~min}^{-1} \\
0 \mathrm{~min}^{-1}\end{array}$ \\
\hline
\end{tabular}

and can be used to study other first-passage processes under confinement.

We hope that our findings may encourage future studies comparing capture mechanisms in different organisms with varying spindle sizes. This may test our basic hypothesis that the physical advantages of one mechanism over others may drive their selection.

\section{ACKNOWLEDGMENTS}

A.N. acknowledges IRCC at IIT Bombay, India, and Science and Engineering Research Board (SERB), India (Project No. ECR/2016/001967) for financial support. I.N. thanks IIT Bombay for Institute Ph.D. fellowship. D.D. and A.N. acknowledge MPIPKS, Dresden, for kind hospitality and use of their computational facility. I.N. acknowledges the High Performance Computing Facility at IIT Bombay.

\section{APPENDIX A: SIMULATION METHOD OF THE MODELS}

Here we first discuss the computational models to study the attachment of MTs to the $\mathrm{KC}$ by RD and $\mathrm{S} \& \mathrm{C}$. The $\mathrm{KC}$ is assumed to be a small sphere of radius $a$ which diffuses freely in the nucleoplasm. The nucleus is modeled as a sphere of radius $R$. MTs can nucleate from either of the spindle pole bodies (SPBs). All the parameter values used in our simulations (see Table II) were taken from experimental measurements done at $T=24{ }^{\circ} \mathrm{C}[8]$.

\section{Kinetics of the kinetochore}

For all four models that we study in this paper [see Fig. 1(c) in the main text], the $\mathrm{KC}$ dynamics is identical. The over- damped dynamics of $\mathrm{KC}[8,61]$ is given by

$$
\frac{d \mathbf{r}_{K C}}{d t}=\sqrt{2 D_{K C}} \boldsymbol{\xi}_{K C}(t) .
$$

Here, $\mathbf{r}_{K C}$ is the position vector and $D_{K C}$ is the translational diffusion coefficient of the $\mathrm{KC}$. The components of $\boldsymbol{\xi}_{K C}(t)$ are Gaussian white noise with mean zero and delta correlations $\left\langle\xi_{i}(t) \xi_{j}\left(t^{\prime}\right)\right\rangle=\delta_{i, j} \delta\left(t-t^{\prime}\right)$ with $i, j=x_{K C}, y_{K C}, z_{K C}$. The initial position of the $\mathrm{KC}$ is uniformly distributed inside a small subsphere around the center of radius $0.3 \mu \mathrm{m}$. At the nuclear boundary, the reflecting boundary condition is applied along the radius vector joining the $\mathrm{KC}$ and the center of the nuclear sphere.

\section{Langevin simulation for rotational diffusion of MTs}

In this process, all the MTs are assumed to have fixed lengths $L_{M T}$, pivoted at either of the SPBs [Fig. 1(c) in the main text]. In the overdamped limit, the equation of motion for the MT in spherical polar coordinates is given by [8]

$$
\begin{aligned}
\frac{d \theta_{M T}}{d t} & =D_{M T} \frac{\cos \theta_{M T}}{\sin \theta_{M T}}+\sqrt{2 D_{M T}} \xi_{\theta_{M T}}(t), \\
\frac{d \phi_{M T}}{d t} & =\frac{\sqrt{2 D_{M T}}}{\sin \theta_{M T}} \xi_{\phi_{M T}}(t) .
\end{aligned}
$$

Here, $D_{M T}$ is the angular diffusion coefficient of the MTs. Similar to $\xi_{K C}$, here $\xi_{M T}=\left(\xi_{\theta_{M T}}, \xi_{\phi_{M T}}\right)$ is a Gaussian white noise with mean zero and delta correlations. The initial orientation of the MTs pivoted at the SPBs are distributed uniformly inside the nuclear envelope. Since MT length $r_{M T}=$ $L_{M T}$ is a constant, the tip of a MT is always constrained to diffuse on a portion of a spherical surface of radius 
$L_{M T}$, with $0 \leqslant \theta_{M T} \leqslant \theta_{M T}^{\max }$ and $0 \leqslant \phi_{M T} \leqslant 2 \pi$. Here $\theta_{M T}^{\max }=$ $\left.\cos ^{-1}\left(L_{M T} / 2 R\right)\right]$. Reflecting boundary conditions are applied when the MT tip hits the boundary.

\section{Langevin-Gillespie hybrid algorithm for search and capture}

The search-and-capture S\&C mechanism involves dynamic instability of MTs [10,12]. Experiments [8], however, showed that the MTs exhibit a state of pause (P) before switching from the growth $(\mathbf{G})$ to the shrink (S) state (also adapted in [17]). To compare the relative efficiency, here we study different models of S\&C both with or without the $\mathbf{P}$ state. MTs have a tubular structure consisting of typically 13 protofilaments [1]. Thus when 13 tubulin dimers are added (or subtracted), the length of a MT $r_{M T}$ increases (or decreases) by a length of $8 \mathrm{~nm}$. Since we do not have explicit protofilaments in our models, we take care of this by choosing the effective subunit length of each dimer to be $8 / 13 \mathrm{~nm}$ in simulations, as has been done in earlier works [62-64]. In all our dynamic instability models, each MT starts out in a random direction with a "seed" length $l_{\text {min }}$ of 20 dimers (i.e., $20 \times 8 / 13 \approx 12 \mathrm{~nm}$ ) and grows by adding subunits with rate $r_{+}$. In $\mathbf{S}$ state, a MT shrinks back to the cutoff length $l_{\min }$ by losing subunits with rate $r_{-}$. Subsequently, the MT switches back to the $\mathbf{G}$ state, choosing a new random direction.

To simulate our models, we use a combination of Langevin dynamics and kinetic Monte Carlo. The $\mathrm{KC}$ position is updated using the Langevin equation [Eq. (A1)], whereas the dynamic instability of a MT is modeled using the Gillespie algorithm [65].

\section{a. Pure search-and-capture model $(S \& C)$}

This is the standard dynamic instability model where the $\mathbf{P}$ state is ignored [Fig. 1(c) in the main text]. MT switches from the $\mathbf{G}$ to the $\mathbf{S}$ state with the catastrophe frequency $f_{+-}$ keeping its orientation fixed. When a growing MT tip hits the nuclear envelope, it switches to the $\mathbf{S}$ state, keeping its orientation unchanged.

\section{b. Search-and-capture with stationary MTs in pause state $(\mathrm{S} \& \mathrm{C}+\mathrm{P})$}

In this case a MT switches from $\mathbf{G}$ to $\mathbf{P}$ with frequency $f_{+0}$ and $\mathbf{P}$ to $\mathbf{S}$, with frequency $f_{0-}$ keeping its orientation fixed [see Fig. 1(c) in the main text and Table II]. Upon hitting the nuclear envelope, the MTs switch to the $\mathbf{P}$ state.

\section{c. Search and capture with rotational diffusion ( $\mathrm{S} \& \mathrm{C}+\mathrm{RD})$}

This model incorporates all the features observed in the experiments. A MT undergoes both rotational diffusion and dynamic instability (i.e., $\mathbf{G} \rightarrow \mathbf{P} \rightarrow \mathbf{S}$ ) simultaneously [see Figs. 1(b) and 1(c) in the main text]. The state of MT remains unchanged upon hitting the nuclear envelope. In this model, unlike $\mathrm{S} \& \mathrm{C}$ or $\mathrm{S} \& \mathrm{C}+\mathrm{P}$, MTs not only have a Gillespie update due to growth, pause and shrinkage, but also a simultaneous Langevin update due to rotational diffusion following Eqs. (A2) and (A3). But while the Langevin updates of $\mathrm{KC}$ and $\mathrm{MT}$ are more frequent and happen at chosen time intervals $\Delta t$, the Gillespie updates for MT growth, pause, and shrinkage events happen occasionally after several such $\Delta t$ intervals.

Since the length $r_{M T}$ varies, we chose a length-dependent angular diffusion constant $\tilde{D}_{M T}=D_{0} / r_{M T}^{3}$ [66-68] for $r_{M T}>$ $L_{M T}$, where $D_{0}=D_{M T} L_{M T}^{3}$ (see Table II). For $r_{M T} \leqslant L_{M T}$, we use $\tilde{D}_{M T}=D_{M T}$-note that having a $r_{M T}^{-3}$ dependence all the way down to small values of $r_{M T}$ would lead to unrealistic values of $\tilde{D}_{M T}$.

\section{Capture conditions}

The positions of the MT tip and the center of mass of the $\mathrm{KC}$ are given by $\mathbf{r}_{M T}=\left(r_{M T}, \theta_{M T}, \phi_{M T}\right)$ and $\mathbf{r}_{K C}=$ $\left(r_{K C}, \theta_{K C}, \phi_{K C}\right)$, respectively. The angle subtended at the SPB is

$$
\gamma=\cos ^{-1}\left(\frac{\mathbf{r}_{K C} \cdot \mathbf{r}_{M T}}{r_{K C} r_{M T}}\right) .
$$

As discussed earlier, the $\mathrm{KC}$ can attach to the tip of the MT or laterally along the body (see Fig. 6). The lateral capture condition is $r_{K C} \cos \gamma \leqslant r_{M T}$ and $r_{K C} \sin \gamma \leqslant a$, or $\left|\mathbf{r}_{K C}-\mathbf{r}_{M T}\right|$ $\leqslant a$. For tip capture (Fig. 6), the capture conditions we use are in conformity with the criterion used by Kalinina et al. in their experiments [8] (see Appendix C).

\section{Effect of finite volume of the microtubules}

In this work, for simplicity, we have ignored the finite volume of the MTs. For a small number of MTs, as in the case for fission yeast, this assumption holds true. As $N$ is increased, the excluded volume effects might become important. Moreover, there is an upper limit on the number of MTs that can be accommodated on the surface of a SPB (see below for details). For S\&C, since MTs growing radially out of the SPB do not explore the volume laterally, this effect has been neglected as in earlier works $[7,15]$. For the RD mechanism, this effect will become important beyond a certain MT number-this may be studied using more sophisticated computational models in future. However, the broad conclusions that we draw from this work, like the relative capture time efficiency of RD over S\&C for different ranges of $N$, are expected to remain unchanged. Moreover, the saturation that we see in $\tau$ with increasing $N$ for RD is expected to occur at a lower value of $N$.

\section{Estimation of maximum number of MTs which may grow out of the surface of SPBS}

The spindle pole body for fission yeast is oblate shaped with diameters $2 d_{1}=0.18 \mu \mathrm{m}$ and $2 d_{2}=0.09 \mu \mathrm{m}$ [69]. Assuming that MTs grow from the surface of SPBs, we may calculate the ratio of the surface area $S_{A}$ of a SPB and the cross-sectional area of a MT. This number gives an estimate of the maximum number of MTs that may grow out of a SPB. The area $S_{A}$ of a SPB is calculated by using the following formula [70]:

$$
S_{A}=2 \pi d_{1}^{2}+\pi \frac{d_{2}^{2}}{e} \log \left(\frac{1+e}{1-e}\right) \approx 0.0673 \mu \mathrm{m}^{2} .
$$

Here $e=\left(1-d_{2}^{2} / d_{1}^{2}\right)$ is the eccentricity of the SPB. Noting that the diameter of a MT is $2 d_{M T}=25 \mathrm{~nm}$ [1], the maximum 
number of MTs that can be accommodated on each SPB is

$$
\frac{S_{A} / 2}{\pi d_{M T}^{2}} \approx 69 \text {. }
$$

The factor of half above takes care of the fact that MTs grow from half of the area of a SPB. Finally, considering both the SPBs at the two poles, we obtain a maximum number of $2 \times 69=138$ MTs which may grow within a fission yeast nucleus.

\section{APPENDIX B: METHODS TO ESTIMATE CHARACTERISTIC CAPTURE TIME}

For the randomly sampled first-capture times of $\mathrm{KC}$ by MTs in the models discussed above, the characteristic times $\tau$ are challenging to estimate. We discuss below two different methods for estimating $\tau$ which we have used in this work.

\section{Method 1: Cloning of systems to calculate $S(t)$}

As discussed in the main text, the asymptotic behavior of the survival probability $S(t)$ is independent of the initial positions of the MTs and the KC and behaves as $\lim _{t \rightarrow \infty} S(t) \sim$ $\exp (-t / \tau)$. However, this robust asymptotic exponential tail may appear at very small values of $S(t)$. Standard computational methods often fix a precision like $\sim 10^{-8}$ for $S(t)$ and try to get the tail behavior from large samples of $t$. But however large the sample number is, at such ordinary levels of precision the asymptotic tail may not even appear, and so accurate determination of $\tau$ remains a challenge. However an algorithm, developed earlier in the context of reactiondiffusion systems [52,53], may be very effectively extended to study problems of survival in confined geometries and in particular, to obtain accurate estimates of asymptotic behavior. The main idea of the algorithm is schematically depicted in Fig. 5. At $t=0$, we start with $M$ random realizations of the

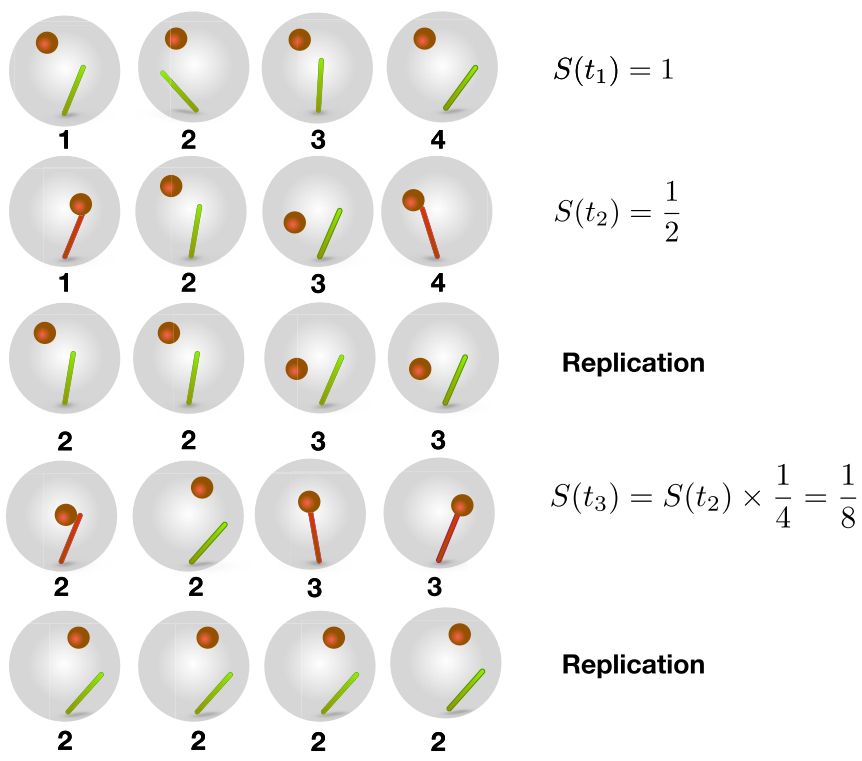

FIG. 5. Illustration of the algorithm of method 1 to calculate the survival probabilities $S(t)$ to high precision. In this figure, $M=4$ and $N=1$. system, each one having $N$ number of MTs and a KC. Initially the survival probability $S(0)=1$. As time evolves, capture happens in some copies, while in the remaining (say $q(t)$ ) copies the KC continues to survive. Thus at any time $t$, we have $S(t)=S(0)[q(t) / M]$. At a time $t=t_{1}$ when $q\left(t_{1}\right) / M=$ $s_{1}$ just becomes $\leqslant 1 / 2$, we replicate the $q\left(t_{1}\right)$ surviving copies to restore the initial ensemble size $M$-this step is referred to as cloning or enrichment [53]. Subsequently, at any $t>t_{1}$, if $q(t)$ are the surviving copies, then $S(t)=s_{1}[q(t) / M]$ until the next enrichment event happens at $t=t_{2}$ when $q\left(t_{2}\right) / M=s_{2}$ becomes just $\leqslant 1 / 2$. This process is iterated many times. For $k$ successive such cloning events $S(t)=s_{1} s_{2} \ldots[q(t) / M]=$ $O\left(1 / 2^{k}\right)$, and thus accuracies like $\sim 10^{-17}$ or even lower can be readily achieved. For such precision of $S(t)$, it is guaranteed that the exponential tail clearly appears, and so $\tau$ is extremely accurately determined. In our simulations, the choice of a large number of realizations, namely, $M=1000$, reduced fluctuations significantly, but the computational cost was fairly large-each $S(t)$ curve for a particular $N$ of each model [see, e.g., Fig. 1(d) in the main text] is generated by running the simulation for $\sim 96 \mathrm{~h}$ using the supercomputing facility at IIT Bombay.

\section{Method 2: Sampling extreme times}

Extreme value statistics deals with extreme (maximum or minimum) deviations of a set of random observations. Consider a set of independent and identically distributed random variables $\left\{t_{1}, t_{2}, \ldots, t_{N_{1}}\right\}$ that are drawn from a parent distribution $F\left(t_{i}\right)$, with $i=1, \ldots, N_{1}$, where

$$
F\left(t_{i}\right) \sim C \exp \left(-t_{i} / \tau\right), \quad \text { for large } t_{i} .
$$

Here $\tau$ is the relaxation constant (characteristic time in our case) and $C$ is a constant. One might be interested in the distribution of the maximum (extreme) value $t_{\max }=\max \left\{t_{i}\right\}$. The cumulative distribution of $t_{\max }$ is

$$
\begin{aligned}
Q(\tilde{t}) & =\operatorname{prob}\left[\tilde{t} \geqslant t_{\max }\right]=\operatorname{prob}\left[\tilde{t} \geqslant t_{1}, \tilde{t} \geqslant t_{2}, \ldots, \tilde{t} \geqslant t_{N_{1}}\right] \\
& =\left[\int_{t_{i}=0}^{\tilde{t}} F\left(t_{i}\right) d t_{i}\right]^{N_{1}}=\left[1-\int_{t_{i}=\tilde{t}}^{\infty} F\left(t_{i}\right) d t_{i}\right]^{N_{1}} .
\end{aligned}
$$

At large $\tilde{t}$, replacing $F\left(t_{i}\right)$ in Eq. (B2) by Eq. (B1) we get

$$
Q(\tilde{t}) \approx[1-C \tau \exp (-\tilde{t} / \tau)]^{N_{1}} .
$$

When $N_{1}$ is large, $Q(\tilde{t})$ in Eq. (B3) can be approximated as follows:

$$
\begin{aligned}
Q(\tilde{t}) & \approx \exp \left(-C N_{1} \tau \exp (-\tilde{t} / \tau)\right) \\
& =\exp \left(-\exp \left[\ln \left(C N_{1} \tau\right)\right] \exp (-\tilde{t} / \tau)\right) \\
& =\exp \left(-\exp \left[-\left(\tilde{t} / \tau-\ln \left(C N_{1} \tau\right)\right)\right]\right),
\end{aligned}
$$

and it finally converges to the cumulative Gumbel distribution $[54,55]$,

$$
Q(\tilde{t}) \approx \exp (-\exp [-(\tilde{t}-\mu) / \tau]),
$$

with $\mu=\tau \ln \left(C N_{1} \tau\right)$. Note that $\mu$ and $\tau$ are nonuniversal constants dependent on the parent distribution $F(t)$.

It is well known that the variance $\sigma^{2}$ of the Gumbel distribution is related to the characteristic value $\tau$ as 
follows [54]:

$$
\tau=\frac{\sqrt{6}}{\pi} \sigma .
$$

The above idea may be used for the purpose of estimating characteristic times of first capture of KC by MTs having the first-passage distribution $F(t)$. Note that the decay constants of the exponential tail of $F(t)$ and survival probability $S(t)$ are the same. In computation or experiment, one needs to first sample $N_{1} \times N_{2}$ different times of capture. The whole data may be divided into $N_{2}$ sets, each having $N_{1}$ sample times. Each such set may provide a $t_{\max }$ such that there would be $N_{2}$ such values. These $t_{\max }$ values may then be used to obtain $\sigma^{2}$, which leads to $\tau$ through Eq. (B6). Table I in the main text show the efficacy of this method.

\section{APPENDIX C: CHARACTERISTIC TIME FOR VARYING MICROTUBULE NUMBER: TIP AND LATERAL CAPTURE}

We study the characteristic time $\tau$ as a function of $N$ for the four different models when the $\mathrm{KC}$ attaches to the tip of a MT. The KC may attach anywhere within $<0.5 \mu \mathrm{m}$ of the MT tip-this was the definition of tip capture used in the experiment [8]. This is shown in Fig. 6(a). We find that the qualitative features are similar to the lateral capture case [shown in Fig. 6(b)]. We see that in both the cases RD model is more efficient than the S\&C model (and also the other two models) for smaller $N$. Similar to the case of lateral capture, here as well we find that $\tau_{R D}$ saturates at large $N$. All other $\tau$ values keep monotonically decreasing. The reason behind the saturation in

(a)

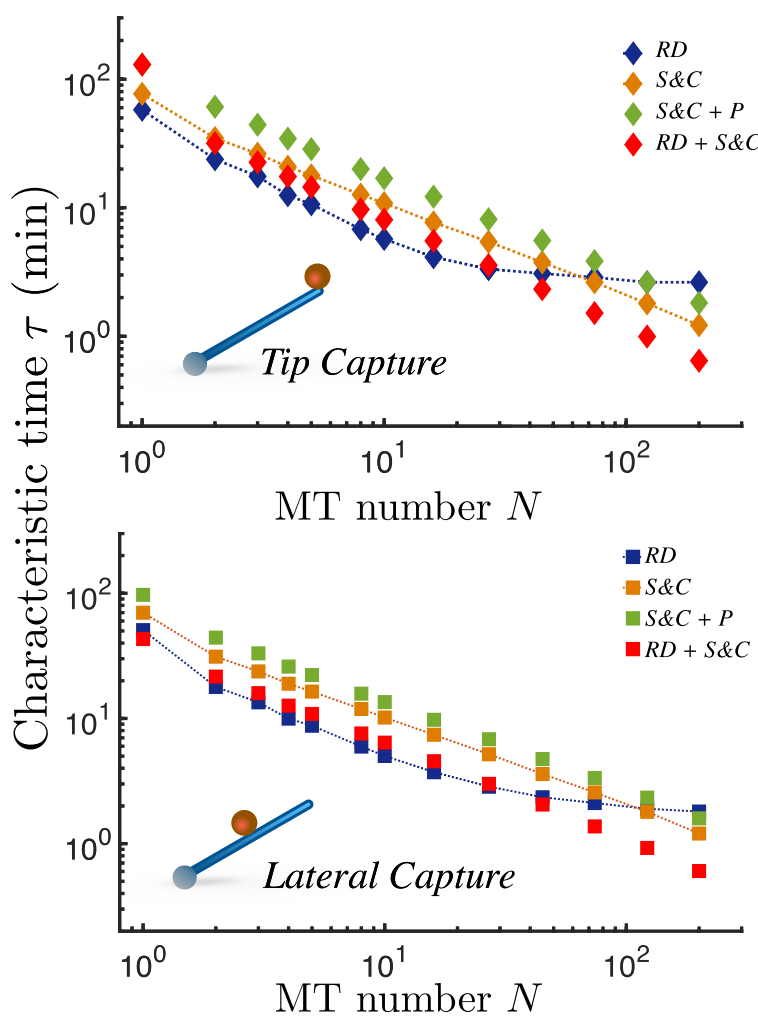

FIG. 6. Characteristic times for (a) tip capture and (b) lateral capture for the four models as a function of $N$.
TABLE III. $\tau_{R D}$ and $\langle t\rangle_{R D}$ compared to other dynamic instability associated models.

\begin{tabular}{llllllll}
\hline \hline & \multicolumn{3}{c}{$\tau_{R D} / \tau_{\text {Model }}$} & & \multicolumn{3}{c}{$\langle t\rangle_{R D} /\langle t\rangle_{\text {Model }}$} \\
\cline { 2 - 4 } \cline { 6 - 8 } Model & $N=1$ & $N=3$ & $N=5$ & & $N=1$ & $N=3$ & $N=5$ \\
\hline RD+S\&C & 1.18 & 0.84 & 0.80 & & 1.10 & 0.74 & 0.74 \\
S\&C & 0.73 & 0.57 & 0.53 & & 0.66 & 0.44 & 0.42 \\
S\&C+P & 0.52 & 0.41 & 0.39 & & 0.43 & 0.30 & 0.27 \\
\hline \hline
\end{tabular}

$\tau_{R D}$ can be explained using the similar argument of geometrical constraint as in the main text. Here, the annular volumes (between radii $L_{M T}$ and $L_{M T}-0.5$ ) on both sides of the nuclear sphere become the region of the capture as $N \rightarrow \infty$. The condition for tip capture is $0 \leqslant\left(r_{M T}-r_{K C} \cos \gamma\right) \leqslant$ 0.5 and $r_{K C} \sin \gamma \leqslant a$, or, $\left|\mathbf{r}_{K C}-\mathbf{r}_{M T}\right| \leqslant a$.

\section{APPENDIX D: EFFICIENCY OF THE RD MODEL WITH RESPECT TO THE OTHER MODELS}

As discussed in the main text, in addition to the mean time $\langle t\rangle$, the characteristic time $\tau$ is a very useful quantity to study. Here, we compare the timescales of four models discussed in the main text to demonstrate the quantitative temporal benefit that the RD mechanism produces. The efficiency of the RD model is expressed in terms of two ratios $\tau_{R D} / \tau_{\text {Model }}$ and $\langle t\rangle_{R D} /\langle t\rangle_{\text {Model }}$ in Table III for MT numbers 1, 3, and 5, respectively. Among the three $N$ values studied below, the efficiency of the pure RD mechanism is most clearly manifested for $N=5$. In Fig. 2 in the main text, we have shown that $\langle t\rangle_{R D}$ is the smallest compared to all the other models for a significant range of $N$. In Table IV, we explicitly write some values of $\langle t\rangle$ for the four different models as a function of $N$ in descending order of temporal efficiency.

\section{APPENDIX E: RATIO OF CHARACTERISTIC AND MEAN TIMESCALES-INDICATOR OF NONEXPONENTIAL FORMS OF $S(t)$}

For a simple exponential function $S(t)=\exp (-t / \tau)$, it is easy to see that the mean time $\langle t\rangle=\int_{0}^{\infty} S(t) d t=\tau$ (characteristic time). But the curves $S(t)$ which we obtain in our study (for example, in Fig. 2 in the main text) have nonexponential functional forms. Nevertheless, they all have exponential tails. The presence of multiple timescales manifest in the fact that $\langle t\rangle \neq \tau$. Thus, the ratio of $\tau /\langle t\rangle$ is an indicator of the nonexponential form of $S(t)$. In Fig. 7, we plot this ratio [by using the data of Fig. 2 in the main text and Fig. 6(b) below] as a function of $N$ for the four different models studied in the

TABLE IV. $\langle t\rangle$ for different models as a function of $N$.

\begin{tabular}{lrccc}
\hline \hline & \multicolumn{4}{c}{$\langle t\rangle(\min )$} \\
\cline { 2 - 5 } Model & $N=5$ & $N=10$ & $N=45$ & $N=122$ \\
\hline RD & 5.38 & 2.29 & 0.73 & 0.58 \\
RD+S\&C & 6.52 & 3.02 & 0.85 & 0.59 \\
S\&C & 11.56 & 5.44 & 1.09 & 0.63 \\
S\&C+P & 17.58 & 8.51 & 1.47 & 0.71 \\
\hline
\end{tabular}




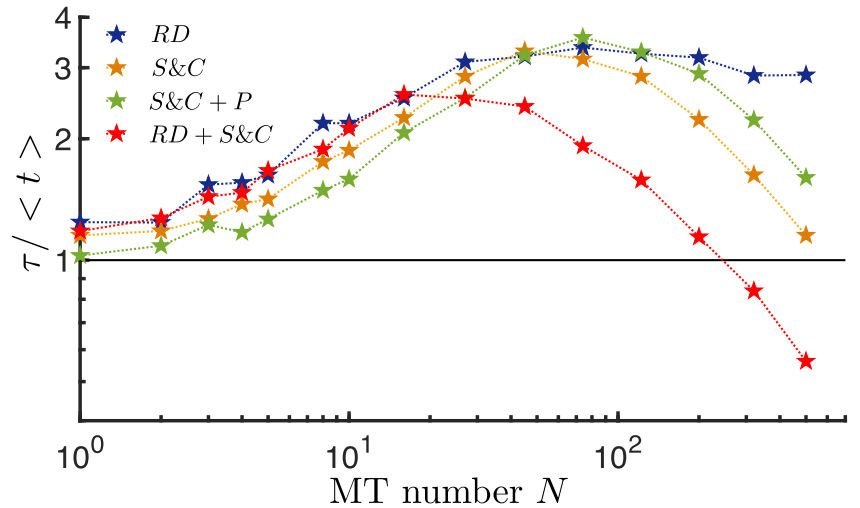

FIG. 7. Ratio of the characteristic and mean capture times for the four models as a function of MT number $N$. The black solid line indicates the case of a simple exponential.

paper. For a few MTs $(<3)$, the values of the ratios are slightly greater than 1, indicating that the corresponding $S(t)$ functions are "almost" single exponentials. But with increasing $N$ there is a strong departure from exponentiality, indicated by the rise of the values of the ratios. In fact, for the RD model even at the largest $N$ that we studied, the ratio stays very different from 1. This compliments our finding that $P(\omega)$ remains bimodal for the RD model for all values of $N$ (hence showing large trajectory-to-trajectory fluctuations in capture times). For the other three models, while $\langle t\rangle$ tends to saturate at large $N$ (see Fig. 2 in the main text), the characteristic time $\tau$ continues to decrease [Fig. 6(b)]. Consequently, the ratio $\tau /\langle t\rangle$ shows a plunge in Fig. 7 at large $N$.

\section{APPENDIX F: QUASI-TWO-DIMENSIONAL MODEL TO EXPLAIN THE SATURATION OF $\tau$ IN THE RD MODEL}

For large $N$, we have seen the saturation behavior of $\tau_{R D}$ in Fig. 4 in the main text and in Fig. 6(b) above. Here we show that this behavior is generic by studying a toy model analytically and computationally. Consider a circle of radius $b$ inside which a small disk of radius $a$ (like the KC) diffuses freely. $N$ point particles (like the tips of $N$ MTs) diffuse along the diameter (see schematic in Fig. 8) of the circle. As $N \rightarrow \infty$ the entire diameter becomes an absorbing line (see schematic in Fig. 8). We perform simulations for finite $N$, and the data is plotted in Fig. 8 with green symbols. We have performed an analytical calculation for $\tau$ which yields $\tau_{\text {sat }} \approx 3.10$ [arbitrary (arb.) unit]; the latter is represented by a black solid line in Fig. 8. Convergence of the computational values of $\tau$ to the analytical value of $\tau_{\text {sat }}$ for $N>10$ is clearly visible. The analytical calculation is discussed below.

\section{Analytical estimate of $\tau_{\text {sat }}$ for the quasi-two-dimensional model}

Using the backward Fokker-Planck equation [61], we analytically estimate here the $\tau_{\text {sat }}$ value for the quasi-2D model in the limit of $N \rightarrow \infty$ (schematic in Fig. 8). The disk of radius $a$ ( similar to the $\mathrm{KC}$ ) diffuses inside a semicircle of radius $b$. The diameter of the semicircle is an absorbing boundary while the

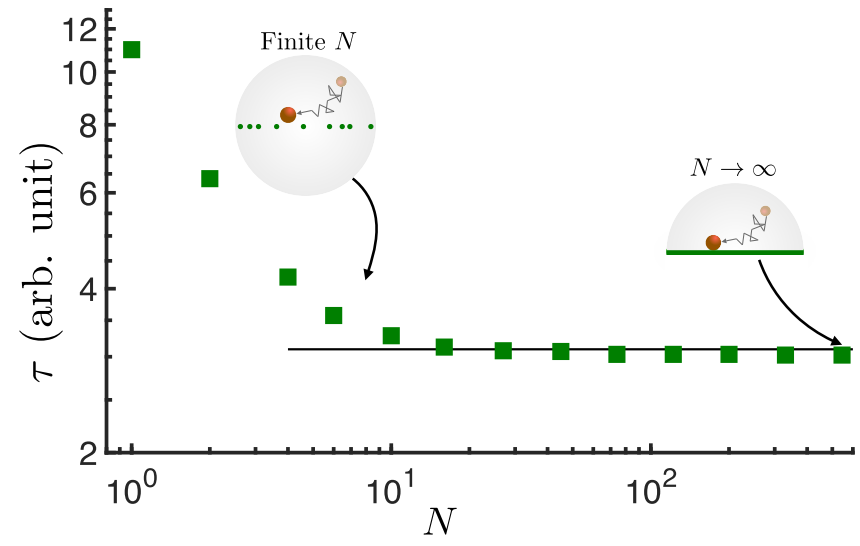

FIG. 8. A toy model to explain the saturation in $\tau$. A disk diffuses inside a circle and can get captured along the diameter where noninteracting particles diffuse along the diameter (see schematic for finite $N$ ). The limiting case here is an absorbing line along the diameter of the circle, which is realized for $N \rightarrow \infty$ (shown schematically). The characteristic time $\tau$ for the toy model (green filled square) shows saturation with $N$. The $\tau_{\text {sat }} \approx 3.10$ (arb. unit) (black line) is obtained from the analytical calculation discussed below.

semicircular arc is a reflecting boundary. For the simplest case where a disk is assumed to be as a point particle $(a=0)$, the backward Fokker-Planck equation for the survival probability $S(r, \theta, t)$ is given by

$$
\frac{\partial S}{\partial t}=D \nabla^{2} S
$$

where $\nabla^{2}$ is the two-dimensional Laplacian operator in polar coordinates. $S(r, \theta, t)$ is the survival probability of the particle up to time $t$ starting from the initial position $(r, \theta)$. Here $D$ is the diffusion coefficient of the disk. We solve Eq. (F1) using a separation of variables. Substituting $S(r, \theta, t)=R(r) \Theta(\theta) T(t)$ in the above equation we get

$$
\frac{1}{D T} \frac{d T}{d t}=\frac{1}{R} \frac{d^{2} R}{d R^{2}}+\frac{1}{R r} \frac{d R}{d r}+\frac{1}{r^{2} \Theta} \frac{d^{2} \Theta}{d \theta^{2}} .
$$

Equating the left-hand side to a constant value $-k^{2}$, we get the $T(t)$ solution as

$$
T(t)=T_{0} \exp \left(-k^{2} D t\right)
$$

and the above equation becomes

$$
\frac{r^{2}}{R} \frac{d^{2} R}{d r^{2}}+\frac{r}{R} \frac{d R}{d r}+k^{2} r^{2}=-\frac{1}{\Theta} \frac{d^{2} \Theta}{d \theta^{2}} .
$$

Equating the right-hand side to $m^{2}$ and applying the absorbing boundary condition $\Theta(\theta)=0$ at $\theta=0$ and $\pi$, we get the solution of the angular part as

$$
\Theta(\theta)=A \sin (m \theta),
$$

where $m$ can be any integer value. Finally, the radial part of Eq. (F3) gives the solution in terms of Bessel functions of order $m$,

$$
R(r)=A_{m} J_{m}(k r)+B_{m} Y_{m}(k r) .
$$


At $r=0$, the radial function should vanish due to the absorbing boundary condition. Since $Y_{m}(k r)$ diverges as $r \rightarrow 0$, we drop that term. The above relation is true for any positive integer $m$. The reflecting boundary condition leads to the vanishing of the first derivative of $R(r)$ at $r=b$, i.e., $\left.\frac{d R}{d r}\right|_{r \rightarrow b}=0$. Using Eq. (F5) and the reflecting boundary condition we obtain [71]

$$
\left.\frac{d J_{m}(k r)}{d r}\right|_{r \rightarrow b}=\frac{m}{b} J_{m}(k b)-k J_{m+1}(k b)=0 .
$$

From Eq. (F2) the characteristic time $\tau_{\text {sat }}=1 / k_{1}^{2} D$, where $k_{1}$ is the smallest positive value of $k$ satisfying Eq. (F6), corresponding to $m=1$. Assuming $k_{1} b$ is a small number, we approximate $J_{m}(k b) \approx \frac{(k b)^{m}}{m ! 2^{m}}$ to finally obtain

$$
k_{1} \approx \frac{2}{b}
$$

Since in our simulation the disk has a finite radius $a$ and the capture happens as the periphery of the disk touches the diameter, we need to replace the radius $b$ in Eq. (F7) by an effective radius $\tilde{b}$. We use a geometrical approximation $\tilde{b} \approx \sqrt{R^{2}-4 a^{2}}-a$. For $b=4.0, a=0.4$, and $D=1$, we get $\tau_{\text {sat }}=\tilde{b}^{2} / 4 D \approx 3.10$ (arb. unit). We further confirm this value by directly simulating the case of $N \rightarrow \infty$ and find a numerical value of $\tau_{\text {sat }}=3.02$ (arb. unit), which is in good agreement with the analytical approximation of 3.10 (arb. unit).
[1] B. Alberts, D. Bray, K. Hopkin, A. Johnson, J. Lewis, M. Raff, K. Roberts, and P. Walter, Essential Cell Biology (Garland Science, New York, 2013).

[2] T. Wittmann, A. Hyman, and A. Desai, Nat. Cell Biol. 3, E28 (2001).

[3] N. Pavin and I. M. Tolić, Annu. Rev. Biophys. 45, 279 (2016).

[4] I. M. Cheeseman and A. Desai, Nat. Rev. Mol. Cell Biol. 9, 33 (2008).

[5] R. E. Carazo-Salas, O. J. Gruss, I. W. Mattaj, and E. Karsenti, Nat. Cell Biol. 3, 228 (2001).

[6] M. J. Sagolla, S. Uzawa, and W. Z. Cande, J. Cell Sci. 116, 4891 (2003).

[7] R. Paul, R. Wollman, W. T. Silkworth, I. K. Nardi, D. Cimini, and A. Mogilner, Proc. Natl. Acad. Sci. USA 106, 15708 (2009).

[8] I. Kalinina, A. Nandi, P. Delivani, M. R. Chacón, A. H. Klemm, D. Ramunno-Johnson, A. Krull, B. Lindner, N. Pavin, and I. M. Tolić-Nørrelykke, Nat. Cell Biol. 15, 82 (2013).

[9] N. Pavin and I. M. Tolić-Nørrelykke, Syst. Synth. Biol. 8, 179 (2014).

[10] T. Mitchison and M. Kirschner, Nature (London) 312, 237 (1984).

[11] M. Kirschner and T. Mitchison, Cell 45, 329 (1986).

[12] T. E. Holy and S. Leibler, Proc. Natl. Acad. Sci. USA 91, 5682 (1994).

[13] T. L. Hill, Linear Aggregation Theory in Cell Biology (Springer Science \& Business Media, New York, 2012).

[14] T. L. Hill, Proc. Natl. Acad. Sci. USA 82, 4404 (1985).

[15] R. Wollman, E. Cytrynbaum, J. Jones, T. Meyer, J. M. Scholey, and A. Mogilner, Curr. Biol. 15, 828 (2005).

[16] M. Gopalakrishnan and B. S. Govindan, Bull. Math. Biol. 73, 2483 (2011).

[17] R. Blackwell, O. Sweezy-Schindler, C. Edelmaier, Z. R. Gergely, P. J. Flynn, S. Montes, A. Crapo, A. Doostan, J. R. McIntosh, M. A. Glaser et al., Biophys. J. 112, 552 (2017).

[18] P. L. Witt, H. Ris, and G. G. Borisy, Chromosoma 81, 483 (1980).

[19] P. Kalab, R. T. Pu, and M. Dasso, Curr. Biol. 9, 481 (1999).

[20] P. Askjaer, V. Galy, E. Hannak, and I. W. Mattaj, Mol. Biol. Cell 13, 4355 (2002).

[21] H. Maiato, C. L. Rieder, and A. Khodjakov, J. Cell Biol. 167, 831 (2004).
[22] M. E. Janson, T. G. Setty, A. Paoletti, and P. Tran, J. Cell Biol. 169, 297 (2005).

[23] K. S. Burbank, A. C. Groen, Z. E. Perlman, D. S. Fisher, and T. J. Mitchison, J. Cell Biol. 175, 369 (2006).

[24] N. M. Mahoney, G. Goshima, A. D. Douglass, and R. D. Vale, Curr. Biol. 16, 564 (2006).

[25] U. S. Tulu, C. Fagerstrom, N. P. Ferenz, and P. Wadsworth, Curr. Biol. 16, 536 (2006).

[26] E. Kitamura, K. Tanaka, S. Komoto, Y. Kitamura, C. Antony, and T. U. Tanaka, Dev. Cell 18, 248 (2010).

[27] V. Magidson, C. B. O'Connell, J. Lončarek, R. Paul, A. Mogilner, and A. Khodjakov, Cell 146, 555 (2011).

[28] S. Petry, A. C. Groen, K. Ishihara, T. J. Mitchison, and R. D. Vale, Cell 152, 768 (2013).

[29] V. Magidson, R. Paul, N. Yang, J. G. Ault, C. B. O'Connell, I. Tikhonenko, B. F. McEwen, A. Mogilner, and A. Khodjakov, Nat. Cell Biol. 17, 1134 (2015).

[30] F. Decker, D. Oriola, B. Dalton, and J. Brugués, Elife 7, e31149 (2018).

[31] N. Van Kampen, J. Stat. Phys. 70, 15 (1993).

[32] O. Bénichou, C. Chevalier, J. Klafter, B. Meyer, and R. Voituriez, Nat. Chem. 2, 472 (2010).

[33] O. Bénichou and R. Voituriez, Phys. Rep. 539, 225 (2014).

[34] A. Godec and R. Metzler, Phys. Rev. X 6, 041037 (2016).

[35] A. Godec and R. Metzler, Sci. Rep. 6, 20349 (2016).

[36] D. Hartich and A. Godec, New J. Phys. 20, 112002 (2018).

[37] P. Krapivsky and S. Redner, J. Phys. A: Math. Gen. 29, 5347 (1996).

[38] S. Redner and P. Krapivsky, Am. J. Phys. 67, 1277 (1999).

[39] A. J. Bray, S. N. Majumdar, and G. Schehr, Adv. Phys. 62, 225 (2013).

[40] G. Cojoc, A.-M. Florescu, A. Krull, A. H. Klemm, N. Pavin, F. Jülicher, and I. M. Tolić, Sci. Rep. 6, 25736 (2016).

[41] R. Blackwell, C. Edelmaier, O. Sweezy-Schindler, A. Lamson, Z. R. Gergely, E. O'Toole, A. Crapo, L. E. Hough, J. R. McIntosh, M. A. Glaser et al., Sci. Adv. 3, e1601603 (2017).

[42] L. Winters, I. Ban, M. Prelogović, I. Kalinina, N. Pavin, and I. M. Tolić, BMC Biol. 17, 42 (2019).

[43] A. R. Lamson, C. J. Edelmaier, M. A. Glaser, and M. D. Betterton, Biophys. J. 116, 1719 (2019). 
[44] C. J. Edelmaier, A. R. Lamson, Z. R. Gergely, S. Ansari, R. Blackwell, J. R. McIntosh, M. A. Glaser, and M. D. Betterton, bioRXiv 649913.

[45] R. Ding, K. L. McDonald, and J. R. McIntosh, J. Cell Biol. 120, 141 (1993).

[46] B. F. McEwen, G. K. Chan, B. Zubrowski, M. S. Savoian, M. T. Sauer, and T. J. Yen, Mol. Biol. Cell 12, 2776 (2001).

[47] A. Sarkar, H. Rieger, and R. Paul, Biophys. J. 116, 2079 (2019).

[48] H. van Beijeren, J. Stat. Phys. 110, 1397 (2003).

[49] D. Hartich and A. Godec, J. Stat. Mech. (2019) 024002.

[50] G. Oshanin, O. Vasilyev, P. Krapivsky, and J. Klafter, Proc. Natl. Acad. Sci. USA 106, 13696 (2009).

[51] S. Redner, A Guide to First-Passage Processes (Cambridge University Press, Cambridge, UK, 2001).

[52] V. Mehra and P. Grassberger, Phys. Rev. E 65, 050101(R) (2002).

[53] P. Grassberger and W. Nadler, in Computational Statistical Physics (Springer, New York, 2002), pp. 169-190.

[54] E. Castillo, A. S. Hadi, N. Balakrishnan, and J.-M. Sarabia, Extreme Value and Related Models in Engineering and Science Applications (John Wiley \& Sons, New York, 2005).

[55] E. J. Gumbel, Statistics of Extremes (Courier Corporation, North Chelmsford, MA, 2012).

[56] C. Mejia-Monasterio, G. Oshanin, and G. Schehr, Phys. Rev. E 84, 035203(R) (2011).
[57] C. Mejia-Monasterio, G. Oshanin, and G. Schehr, J. Stat. Mech. (2011) P06022.

[58] T. G. Mattos, C. Mejia-Monasterio, R. Metzler, and G. Oshanin, Phys. Rev. E 86, 031143 (2012).

[59] T. G. Mattos, C. Mejia-Monasterio, R. Metzler, G. Oshanin, and G. Schehr, in First-Passage Phenomena and Their Applications (World Scientific, Singapore, 2014), pp. 203-225.

[60] I. Nayak, A. Nandi, and D. Das (unpublished).

[61] C. Gardiner, Handbook of Stochastic Methods for Physics, Chemistry, and the Natural Sciences (Springer-Verlag, Berlin, 1985).

[62] D. Das, D. Das, and R. Padinhateeri, New J. Phys. 16, 063032 (2014).

[63] R. Padinhateeri, A. B. Kolomeisky, and D. Lacoste, Biophys. J. 102, 1274 (2012).

[64] J. Aparna, R. Padinhateeri, and D. Das, Sci. Rep. 7, 45747 (2017).

[65] D. T. Gillespie, J. Comput. Phys. 22, 403 (1976).

[66] M. Doi and S. F. Edwards, The Theory of Polymer Dynamics (Oxford University Press, Oxford, UK, 1988), Vol. 73.

[67] H. Löwen, Phys. Rev. E 50, 1232 (1994).

[68] S. Broersma, J. Chem. Phys. 32, 1626 (1960).

[69] J.-Q. Wu and T. D. Pollard, Science 310, 310 (2005).

[70] W. Beyer, CRC Standard Mathematical Tables (CRC Press, Boca Raton, FL, 1987).

[71] G. F. Simmons, Differential Equations with Applications and Historical Notes (CRC Press, Boca Raton, FL, 2016). 